\title{
Devenir des lipides au cours de la clarification du lactosérum
}

\author{
C Théodet, G Gandemer* \\ Laboratoire d'étude des interactions des molécules alimentaires, INRA, \\ BP 527, 44026 Nantes Cedex 03, France
}

(Reçu le 6 janvier 1994; accepté le 11 avril 1994)

Résumé - Cette étude a pour objet de caractériser les lipides du lactosérum et de 2 coproduits issus de sa délipidation par agrégation thermocalcique des lipoprotéines. À partir d'un lactosérum contenant $0,7 \%$ de lipides, il est possible d'obtenir un microfiltrat pratiquement dépourvu de lipides $(0,07 \%)$ et un précipité enrichi en lipides (2,9\%). Les extraits lipidiques du lactosérum et de ces 2 coproduits présentent des caractéristiques très voisines (rapport triglycérides/phospholipides, compositions en acides gras des triglycérides et des phospholipides). Ces résultats indiquent que le procédé n'est pas sélectif vis-à-vis des lipides. Les phospholipides du lactosérum comportent $34 \%$ de phosphatidyl-éthanolamine, $31 \%$ de phosphatidyl-choline et $15 \%$ de sphingomyéline, $12 \%$ de phosphatidyl-inositol et $8 \%$ de phosphatidyl-sérine. Ces phospholipides sont pauvres en acides gras polyinsaturés quelle que soit la classe considérée (3 à 17\%).

lipide / phospholipide / lactosérum / coproduit / délipidation

Summary - Fate of lipids during whey defatting process. The aim of the study was to investigate the lipid composition of a whey and two byproducts from its defatting by thermocalcic aggregation of lipoproteins. From a whey containing $0.7 \%$ of lipids, two fractions were separated: a microfiltrate with very low lipid content $(0.07 \%)$ and a precipitate containing $2.9 \%$ of lipids. The lipid extracts of these whey fractions show similar lipid composition (triglyceride /phospholipid ratio, triglyceride and phospholipid fatty acid composition). These results indicate that the process exhibits no selective effect on lipid. The whey phospholipids contain $34 \%$ of phosphatidyl-ethanolamine, $31 \%$ of phosphatidyl-choline and $15 \%$ of sphingomyelin, $12 \%$ of phosphatidyl-inositol and $8 \%$ of phosphatidyl-serine. Whatever the phospholipid class, the polyunsaturated fatty acid proportion is low (3 to 17\%).

lipid / phospholipid / whey / byproduct / defatting

\footnotetext{
${ }^{\star}$ Correspondance et tirés à part
} 


\section{INTRODUCTION}

Les lipides ne constituent qu'une faible proportion de la matière sèche des lactosérums (Cerbulis et al, 1972; Fauquant et al, 1985 b). Toutefois, leur présence s'avère préjudiciable à la valorisation des protéines (Maubois et al, 1987). En effet, ils se concentrent avec les protéines et s'adsorbent sur les membranes de microfiltration et sur les résines échangeuses d'ions altérant leurs performances. Leur élimination est donc un préalable indispensable à la production de concentrés à haute teneur en protéines et au fractionnement des protéines du lactosérum. C'est pourquoi Fauquant et al (1985 a et b) ont développé un procédé industriel qui permet de précipiter les lipides en provoquant leur agrégation avec les protéines en présence de calcium à $\mathrm{pH} 7,3$ sous laction d'un léger traitement thermique. Par microfiltration, il est possible de séparer un microfiltrat pratiquement exempt de lipides et un rétentat enrichi en lipides appelé concentré lipoprotéique. Cependant, ce procédé reste largement empirique. Au moment où nous avons entrepris cette étude, aucun travail ne permettait de connaître la nature des lipides du lactosérum et leur devenir au cours de la clarification. Très récemment, Baumy et al (1990) ont établi que les extraits lipidiques totaux des lactosérums et des concentrés lipoprotéiques contiennent environ un tiers de phospholipides et deux tiers de triglycérides. Toutefois, la caractérisation de la fraction lipidique du lactosérum et de ses coproduits n'était pas assez précise pour qu'il soit possible de se prononcer sur les mécanismes mis en jeu lors de la précipitation des lipides. Pour leur part, Ducruet et al (1990) ont déterminé en détail la composition lipidique d'un concentré lipoprotéique. Leurs résultats plaident en faveur d'une nette discrimination des lipides au cours du procédé. En effet, ces auteurs indiquent que plus de $30 \%$ de lipides polaires du concentré lipoprotéique seraient des céramides alors que ces composés sont peu abondants dans le lait $(3$ à $7 \%$ des lipides polaires) (Morrison, 1970; Christie et al, 1987). Á l'inverse, la phosphatidyl-éthanolamine serait peu abondante dans le concentré alors que ce phospholipide est un constituant majeur des lipides polaires du lait (8 à $9 \%$ contre 25 à $30 \%$ ) (Jensen, 1973 ; Christie et al, 1987). Malheureusement. l'absence totale de données sur la composition lipidique du lactosérum utilisé pour produire le concentré interdit de conclure de manière définitive à une sélectivité du procédé de clarification du lactosérum vis-à-vis des lipides.

Cette étude a pour objet de suivre le devenir des lipides du lactosérum au cours de la clarification pour tenter d'en comprendre le mécanisme en comparant la composition lipidique d'un lactosérum et des 2 coproduits issus de sa clarification suivant le procédé de Fauquant et al (1985 $a$ et b). Ce travail a été complété par une caractérisation précise des phospholipides du lactosérum et du concentré lipoprotéique pour en évaluer les possibilités de valorisation.

\section{MATÉRIEL ET MÉTHODES}

\section{Origine des matières premières}

Un lactosérum issu de la fabrication d'emmental a subi une clarification par précipitation thermocalcique des "phospholipoprotéines" selon le procédé de Fauquant et al (1985 a,b). Une microfiltration tangentielle a permis la séparation du précipité (concentré lipoprotéique) de la fraction soluble (microfiltrat); 25 I de lactosérum, de concentré lipoprotéique et de microfiltrat ont été collectés. Les 3 fractions n'ont été ni déminéralisées ni délactosées. Pour permettre leur conservation, elles ont été additionnées d'azide de 
sodium $(0,02 \%)$ et lyophilisées dans un appareil de laboratoire Virtis. Les lyophilisats sont conservés dans des flacons opaques à $4^{\circ} \mathrm{C}$.

\section{Dosage des constituants non lipidiques}

\section{Cendres}

La teneur en cendres est déterminée par minéralisation de $6 \mathrm{~g}$ de matière sèche à $600^{\circ} \mathrm{C}$ pendant une nuit. Elle est exprimée en $\mathrm{g} / 100 \mathrm{~g}$ de matière sèche.

\section{Azote}

L'azote total est quantifié selon la méthode Kjeldahl. La teneur en protéines est calculée en multipliant le taux d'azote par 6,38. Elle est exprimée en $\mathrm{g} / 100 \mathrm{~g}$ de matière sèche.

\section{Lactose et oses libres}

Les oses ont été quantifiés par chromatographie en phase gazeuse de leurs dérivés acétates d'alditol. Ces dérivés sont préparés suivant la méthode de Blakeney et al (1983). Le mode opératoire a été appliqué directement aux échantillons pour quantifier les oses libres. Une seconde opération a été effectuée sur les échantillons préalablement hydrolysés pendant $2 \mathrm{~h}$ à $100^{\circ} \mathrm{C}$ par l'acide sulfurique $2 \mathrm{~N}$ pour quantifier les oses totaux. La proportion de lactose est calculée par différence entre ces 2 mesures. L'analyse est réalisée sur une colonne remplie $(0,22 \mathrm{~cm} \times 180$ $\mathrm{cm}$ ) contenant une phase stationnaire polaire (SP-2340). Elle est placée dans un chromatographe Girdel muni d'un détecteur à ionisation de flamme et d'un injecteur. La température du four est maintenue constante à $225^{\circ} \mathrm{C}$ et celles de l'injecteur et du détecteur à $250^{\circ} \mathrm{C}$. La pression du gaz vecteur (azote) est de 1,8 bar, L'intégration des pics est réalisée à l'aide d'un intégrateur Shimatzu (CR3A). Les oses sont quantifiés par rapport à l'inositol ajouté comme étalon interne. Les résultats sont exprimés en $\mathrm{g} / 100 \mathrm{~g}$ de matière sèche.

\section{Caractérisation de la fraction lipidique}

\section{Extraction des lipides}

Elle est réalisée suivant la méthode de Clark et al (1982). Les avantages de cette méthode ont été décrits dans un travail précédent, qui avait permis de comparer 5 méthodes d'extraction des lipides du lactosérum et d'un concentré lipoprotéique (Théodet et Gandemer, 1991)

Cinq à $10 \mathrm{~g}$ de lyophilisat de lactosérum, de microfiltrat ou de concentré lipoprotéique sont préalablement réhydratés pour permettre l'extraction quantitative des lipides. Cette opération est réalisée en ajoutant $20 \mathrm{ml}$ d'eau à $10 \mathrm{~g}$ de lyophilisat, puis en agitant le mélange pendant environ $2 \mathrm{~h}$, à l'aide d'un agitateur magnétique. Les échantillons sont transvasés dans une ampoule à décanter et additionnés du mélange solvant dichlorométhane/metthanol $(2 / 1, \mathrm{v} / \mathrm{v})$ à raison de 9 volumes de solvant pour un volume d'échantillon. Après une nuit de décantation à $4^{\circ} \mathrm{C}$, la phase inférieure est soutirée. La phase supérieure est reprise par du mélange dichlorométhane/méthanol $(4 / 1, v / v)$ à raison de 8 volumes de solvant pour un volume d'échantillon. Après décantation, la phase inférieure est soutirée. Les 2 phases inférieures sont regroupées et lavées avec une solution saline à $0,6 \%$ de $\mathrm{NaCl}$ ( 1 volume de solution saline pour 5 volumes de solvant). La phase inférieure est filtrée sur sulfate de sodium anhydre. Les solvants sont éliminés à l'aide d'un évaporateur rotatif, puis sous un léger courant d'azote. Les extractions sont effectuées en double. La teneur en lipides est déterminée par pesée et exprimée en $\mathrm{g} / 100 \mathrm{~g}$ de matière sèche.

\section{Fractionnement de l'extrait lipidique total en lipides neutres et polaires}

L'extrait lipidique est fractionné en lipides neutres et polaires sur cartouche de silice (Sep-pack, Waters SA) selon la méthode de Juaneda et Rocquelin (1985). Les lipides neutres sont élués par du chloroforme, puis sont quantifiés par pesée. Les phospholipides sont élués par le méthanol ; ils sont quantifiés par dosage du phosphore directement dans l'extrait lipidique total selon la méthode de Bartlett (1959). La teneur en phospholipides est calculée en multi- 
pliant le taux de phosphore lipidique par le coefficient 25.

Les teneurs en lipides neutres et phospholipides sont exprimées en $\mathrm{g} / 100 \mathrm{~g}$ de matière sèche.

\section{Fractionnement des phospholipides en classes}

Le fractionnement en classes des phospholipides est réalisé par chromatographie liquide haute pression. L'appareil comprend 2 pompes, un amortisseur de pulsation, une chambre de mélange et une vanne d'injection théodyne équipée d'une boucle de $20 \mu \mathrm{l}$. II est piloté par un Apple Ile. Les colonnes sont en acier et remplies de silice (Lichrospher, $5 \mu \mathrm{m}$ ). Les phospholipides sont détectés à l'aide d'un détecteur à diffusion de lumière (DDL 10, Cunow) relié à un système d'intégration Nelson. Les conditions optimales de fonctionnement du détecteur pour la quantification des phospholipides ont été déterminées antérieurement (Leseigneur et al, 1989). Elles sont les suivantes: pression du gaz de nébulisation, 2,2 bars ; pression du gaz auxiliaire, 0,5 bar; température de nébulisation du solvant $40^{\circ} \mathrm{C}$. Dans ces conditions opératoires, la réponse du détecteur est une fonction linéaire de la concentration dans une gamme comprise entre 5 et 200 $\mu \mathrm{g}$ de phospholipides par pic.

L'élution des phospholipides fait appel à un gradient de solvants. Les phases mobiles A et B sont composées respectivement de chloroforme pur et du mélange méthanol/eau/ammoniaque/chloroforme (92:5:2:1, v/v/v/v).

La détermination de la composition en phospholipides est réalisée avec une colonne analytique en acier de $25 \mathrm{~cm}$ de long et de $0,4 \mathrm{~mm}$ de diamètre interne contenant de la silice (Superspher, SI-60, $5 \mu \mathrm{m}$ ). L'élution des phospholipides est complète en $20 \mathrm{~min}$. La proportion de solvant $\mathrm{B}$ passe de $0 \%$ à $20 \%$ en 10 min puis de $20 \%$ à $100 \%$ en $10 \mathrm{~min}$. Le débit de la phase mobile est de $1,5 \mathrm{ml} / \mathrm{min}$. Les résultats sont exprimés en pourcentage de la surface des phospholipides totaux ou en $\mathrm{mg} / 100 \mathrm{~g}$ de matière sèche.

Pour déterminer la composition en acides gras de chaque classe de phospholipides, il est nécessaire de disposer d'au moins $0,1 \mathrm{mg}$ de lipides. C'est pourquoi nous avons eu recours à une colonne semi-préparative de $30 \mathrm{~cm}$ de long et de $7,5 \mathrm{~mm}$ de diamètre interne contenant de la silice (Lichrospher, SI-60-5 $\mu \mathrm{m}$ ). Les quantités de phospholipides injectés variaient de 2 à $5 \mathrm{mg}$. Le débit de la phase mobile est de $3,5 \mathrm{ml} / \mathrm{min}$. Les phospholipides sont élués en $60 \mathrm{~min}$. Une vanne placée en sortie de colonne permet de diviser le flux et de collecter environ les trois quarts des phospholipides injectés.

\section{Composition en acides gras}

La composition en acides gras des fractions est déterminée par chromatographie en phase gazeuse de leurs esters isopropyliques ou méthyliques.

Les esters isopropyliques sont préparés selon la méthode de Wolff et Fabien (1989). Cette méthode a été utilisée pour déterminer la composition en acides gras des lipides totaux, neutres et polaires. En effet, ces esters présentent l'avantage de faciliter la quantification des acides gras à chaîne courte puisque ces esters présentent des coefficients de réponse proches de 1 quelle que soit leur longueur de chaîne.

Les phospholipides ne contenant que des acides gras à 10 atomes de carbone et plus, la détermination de leur composition en acides gras ne nécessite pas la préparation d'esters isopropyliques. C'est pourquoi la composition en acides gras des principales classes de phospholipides a été déterminée par chromatographie en phase gazeuse de leurs esters méthyliques, technique classiquement utilisée au laboratoire. Les esters méthyliques sont préparés par transestérification des phospholipides dans le méthanol / $\mathrm{BF}_{3}$ à $14 \%$ suivant la technique de Morrison et Smith (1964).

La chromatographie des esters est réalisée à Yaide d'un chromatographe DANI 6500 (Stang Instruments, France) équipé d'un injecteur PTV et d'un détecteur à ionisation de flamme. II est connecté à un intégrateur ENICA 21 (Delsi Instrument, France). Une colonne capillaire en silice fondue de $30 \mathrm{~m}$ de longueur et $0,32 \mathrm{~mm}$ de diamètre interne contenant une phase stationnaire polaire (polyéthylène glycol) a été utilisée (Superox II, Altech). L'injection est réalisée en mode division $\left(1 / 50^{\mathrm{e}}\right)$. La température du four est maintenue 4 min à $150^{\circ} \mathrm{C}$, puis programmée à $5^{\circ} \mathrm{C} / \mathrm{min}$ jusqu'à $180^{\circ} \mathrm{C}$ où elle est stabilisée jusqu'à la fin de l'analyse. Les températures de linjecteur et du détecteur sont de $250^{\circ} \mathrm{C}$. La pres- 
sion du gaz vecteur en tête de colonne (hydrogène) est de 0,6 bar.

Les esters ont été identifiés par couplage spectrométrie de masse-chromatographie en phase gazeuse (HP 5971A-HP 58901I, HewlettPackard). Les conditions opératoires sont les suivantes : ionisation par impact électronique à 70 $e V$, vitesse d'acquisition des spectres de $1,5 \mathrm{scan} / \mathrm{s}$ sur une gamme de masse de 33 à 400 uma. La composition en acides gras est exprimée en pourcentage des surfaces des esters injectés.

\section{Dosage du cholestérol}

La teneur en cholestérol total est déterminée par chromatographie en phase gazeuse de son dérivé TMS. La technique utilisée s'inspire directement de celle de Mordret et al (1984). Les modifications apportées ont trait à la quantité de lipides mis en oeuvre (réduction à moins de 30 $\mathrm{mg}$ ) et au choix de l'étalon interne (cholestane au lieu du cholestanol). La chromatographie est menée à l'aide d'un chromatographe GIRDEL $300 \mathrm{C}$ muni d'un détecteur à ionisation de flamme et d'un injecteur diviseur (split/splitless). L'analyse est pratiquée sur une colonne capillaire de silice fondue de $10 \mathrm{~m}$ de longueur et $0,32 \mathrm{~mm}$ de diamètre interne contenant une phase stationnaire peu polaire (SE 52, Chrompack). La température du four est maintenue constante à $290^{\circ} \mathrm{C}$, les températures de l'injecteur et du détecteur sont de $300^{\circ} \mathrm{C}$. La pression du gaz vecteur (hydrogène) est de 0,7 bar. Le coefficient de réponse du cholestérol par rapport au cholestane a été évalué à 1,1. La teneur en cholestérol est exprimée en $\mathrm{mg} / 100 \mathrm{~g}$ de matière sèche ou en pourcentage des lipides totaux.

\section{RÉSULTATS}

\section{Comparaison de la composition chimique du lactosérum et de ses 2 coproduits}

Les oses représentent au moins la moitié de l'extrait sec $(52-75 \%)$, quelle que soit la fraction considérée (tableau I). Ce résultat est logique puisque les échantillons ne sont pas délactosés. La recherche d'oses libres n'a pas permis de révéler leur présence dans le lactosérum. En revanche, dans le concentré lipoprotéique, ils représentent $13 \%$ de l'extrait sec. Ce sont du galactose $(8 \%)$, du glucose $(5 \%)$ et de l'arabinose (traces). Leur origine est difficile à expliquer. En effet, le rapport galactose/glucose est de 1,6. Par conséquent, ces oses ne peuvent pas provenir de la seule hydrolyse du lactose. Ils pourraient provenir en partie de l'hydrolyse des glycoprotéines ou des glycolipides.

Les minéraux représentent 8,2 et $10,7 \%$ de la matière sèche du lactosérum et du microfiltrat, respectivement. Le concentré lipoprotéique en contient une proportion

Tableau I. Composition chimique du lactosérum, du microfiltrat et du concentré lipoprotéique (en $\mathrm{g} / 100 \mathrm{~g}$ de matière sèche).

Chemical composition of whey, microfiltrate and lipoprotein precipitate (in g/100 g of dry matter).

Lactosérum

Protéines $(\mathrm{N} \times 6,38)$

Minéraux

Lipides

Protéines/lipides
Microfiltrat

Concentré

lipoprotéique 
nettement plus élevée $(22,4 \%)$. Ce résultat est la conséquence de l'ajout du $\mathrm{CaCl}_{2}$, mais également de la coprécipitation d'une partie des ions phosphate lors du procédé de clarification.

Le taux de protéines du lactosérum $(16,4 \%)$ est intermédiaire entre celui du microfiltrat $(13,7 \%)$ et celui du concentré lipoprotéique $(21,8 \%)$.

À l'aide des données relatives aux volumes de concentré lipoprotéique et de microfiltrat obtenus lors de la clarification de 100 I de lactosérum, nous avons pu effectuer le bilan matière pour chaque constituant (tableau II). $100 \mathrm{I}$ de lactosérum conduisent à la production de $84 \mathrm{I}$ de microfiltrat (6/7 du volume initial) et de 16 I de concentré lipoprotéique (1/7). Le bilan matière montre que le lactose initialement contenu dans le lactosérum se retrouve essentiellement dans le microfiltrat $(85 \%)$, alors que seulement $13 \%$ de ce composé sont retenus dans le concentré lipoprotéique. En effet, le lactose est une molécule à très faible poids moléculaire qui franchit les pores des membranes de microfiltration. Les pertes en lactose au cours du procédé sont minimes ( $2 \%)$. La quantité de protéines précipitées dans le concentré lipoprotéique représente environ $24 \%$ des protéines initialement contenues dans le lactosérum, la majorité des protéines se retrouvant dans le microfiltrat $(70 \%)$. La perte globale en protéines est évaluée à $6 \%$.

Les lipides représentent respectivement $0,7 \%, 0,07 \%$ et $2,9 \%$ de la matière sèche du lactosérum, du microfiltrat et du concentré lipoprotéique. Le rapport protéines/ lipides très élevé (137) dans le microfiltrat montre clairement que le procédé de clarification élimine une grande partie des lipides du lactosérum (tableau 1);74\% des lipides du lactosérum se retrouvent dans le concentré lipoprotéique qui présente un rapport protéines/lipides 3 fois moins élevé que celui observé dans le lactosérum ( 8 au lieu de 23). II faut souligner que $18 \%$ des lipides sont perdus au cours de la clarification.

Les proportions relatives de lipides neutres et de phospholipides dans les extraits lipidiques du lactosérum, du concentré lipoprotéique et du microfiltrat sont très voisines. Les lipides neutres représentent de 58 à $62 \%$ de l'extrait lipidique et les phospholipides de 38 à $42 \%$ (tableau III).

La teneur en cholestérol du lactosérum est de $18 \mathrm{mg} / 100 \mathrm{~g}$ de matière sèche alors que celle du concentré lipoprotéique est de $71 \mathrm{mg} / 100 \mathrm{~g}$. Toutefois, exprimée en $\mathrm{g} / 100 \mathrm{~g}$ de lipides totaux, la proportion de

Tableau II. Devenir des principaux constituants du lactosérum au cours du procédé de clarification. The fate of the main whey components during whey defatting process.

\begin{tabular}{lccc}
\hline & Lactosérum & Microfiltrat & $\begin{array}{c}\text { Concentré } \\
\text { lipoprotéqque } \\
(161)\end{array}$ \\
\hline Matière sèche $(\mathrm{g})$ & $(100 \mathrm{I})$ & $(84 \mathrm{I})$ & 1190 \\
Oses $(\mathrm{g})$ & 6620 & 5564 & 619 \\
Protéines $(\mathrm{N} \times 6,38)(\mathrm{g})$ & 4899 & 4173 & 260 \\
Minéraux $(\mathrm{g})$ & 1086 & 762 & 266 \\
Lipides $(\mathrm{g})$ & 543 & 595 & 34,5 \\
\hline
\end{tabular}


cholestérol dans l'extrait lipidique est comparable dans les deux fractions $(2,5 \%)$ (tableau III). La faible quantité de lipides extraits du microfiltrat n'a pas permis de mesurer le taux de cholestérol de cette fraction.

Les compositions en acides gras de l'extrait lipidique total du lactosérum, du concentré lipoprotéique et du microfiltrat sont comparables (tableau IV). La proportion d'acides gras saturés est élevée $(69,7-72,1 \%)$. Les acides gras à chaîne courte $(4: 0$ à $8: 0)$ représentent $2,4-3,2 \%$, ceux à chaîne moyenne (10:0 à 15:0) 17,8 à $19,6 \%$ et ceux à chaîne longue (16:0 à 24:0) 46,2 à 46,9\%. Les acides gras saturés majeurs sont l'acide palmitique $(29,1$ $32,2 \%)$ et l'acide stéarique $(11,6-14,3 \%)$. II faut souligner la présence de 1,7 à $2,9 \%$ d'acides gras saturés à très longue chaîne $(22,23$, et 24 atomes de carbone). Les acides gras ramifiés caractéristiques des lipides des ruminants sont présents à des taux de $2,4-3,1 \%$. Ce sont principalement les iso et anteiso $15(0,7-1,1 \%)$ et les iso et anteiso $17(1,2-1,7 \%)$, les iso 16 et iso 18 sont présents à des teneurs inférieures à $0,6 \%$. Le taux d'acides gras monoinsaturés est de $24,1-25,3 \%$. L'acide oléique est l'acide gras monoinsaturé le plus abondant $(18,8-19,5)$. II est accompagné de 2,1 à $2,2 \%$ d'acide cis-vaccenique, de $0,6-0,8 \%$ d'acide myristoléique $(14: 1)$ et de 0,9 à $1,6 \%$ d'acide palmitoléique $(16: 1 n-7)$. Les acides gras polyinsaturés sont en quantité faible $(3,8-5,0 \%)$. Ce sont essentiellement de l'acide linoléique $(3,0-4,1 \%)$ et de l'acide linolénique $(0,8-1,0 \%)$. L'acide arachidonique est présent à l'état de traces.

Cette composition en acides gras de l'extrait lipidique total du lactosérum et de ses deux co-produits est voisine de celle des triglycérides (tableau V).

\section{Phospholipides du lactosérum et du concentré lipoprotéique}

Dans le lactosérum et le concentré lipoprotéique, les lipides polaires représentent environ un tiers de l'extrait lipidique total. Ce sont essentiellement des phospholipides. Les céramides ne sont présents qu'en très faible proportion ( $<1 \%$ ). Les proportions relatives des principales classes de phospholipides sont similaires dans le lactosérum et le concentré lipoprotéique (tableau

Tableau III. Composition lipidique du lactosérum, du microfiltrat et du concentré lipoprotéique. Lipid composition of whey, microfiltrate and lipoprotein precipitate.

\begin{tabular}{lccc}
\hline & Lactosérum & Microfiltrat & $\begin{array}{c}\text { Concentré } \\
\text { lipoprotéique }\end{array}$ \\
\hline Lipides totaux $^{1}$ & 730 & 70 & 2900 \\
Lipides neutres (LN) $^{1}$ & 400 & 40 & 1800 \\
Lipides polaires (LP) $^{1}$ & 330 & 30 & 1100 \\
LN/LP 2 $^{\text {Cholestérol 2 }}$ & 1,2 & 1,2 & 1,5 \\
1 & 18 & - & 71 \\
2 & 2,5 & - & 2,5 \\
\hline
\end{tabular}

1: $\mathrm{mg} / 100 \mathrm{~g}$ de matière sèche; 2: pourcentage de l'extrait lipidique total. 
Tableau IV. Composition en acides gras des lipides totaux du lactosérum, du microfiltrat et du concentré lipoprotéique (en \% des esters isopropyliques).

Fatty acid composition of total lipid extract of whey, microfiltrate and lipoprotein precipitate (in \% of isopropyl esters).

Acides gras

Lactosérum

Microfiltrat

Concentré

lipoprotéique

\begin{tabular}{|c|c|c|c|}
\hline $4: 0$ & 0,9 & 1,0 & 1,0 \\
\hline $6: 0$ & 0,7 & 0,6 & 0,4 \\
\hline $8: 0$ & 1,0 & 1,6 & 1,0 \\
\hline 10:0 & 2,2 & 2,8 & 3,1 \\
\hline 12:0 & 3,3 & 3,8 & 3,4 \\
\hline $14: 0$ & 10,8 & 11,4 & 10,6 \\
\hline $15: 0$ & 1,5 & 1,6 & 1,3 \\
\hline $16: 0$ & 29,3 & 32,2 & 29,1 \\
\hline $17: 0$ & 0,8 & 1,2 & 0,8 \\
\hline $18: 0$ & 12,8 & 11,6 & 14,3 \\
\hline $20: 0$ & 0,4 & 0,2 & 0,3 \\
\hline 22:0 & 1,1 & 0,6 & 1,0 \\
\hline 23:0 & 1,0 & 0,6 & 1,2 \\
\hline $24: 0$ & 0,8 & 0,5 & 0,6 \\
\hline Saturés linéaires & 66,6 & 69,7 & 68,1 \\
\hline iso 15 & 0,5 & 0,3 & 0,4 \\
\hline ante iso 15 & 0,7 & 0,4 & 0,6 \\
\hline iso 16 & 0,4 & - & 0,4 \\
\hline iso 17 & 0,7 & 0,7 & 0,6 \\
\hline ante iso 17 & 0,7 & 1,0 & 0,6 \\
\hline iso 18 & 0,1 & - & 0,2 \\
\hline Saturés ramifiés & 3,1 & 2,4 & 2,8 \\
\hline Saturés totaux (S) & 69,7 & 72,1 & 70,9 \\
\hline $14: 1$ & 0,8 & 0,8 & 0,6 \\
\hline $16: 1 n-9$ & 1,4 & 1,0 & 1,4 \\
\hline $16: 1 n-7$ & 1,3 & 0,9 & 1,0 \\
\hline $17: 1$ & 0,1 & - & 0,2 \\
\hline $18: 1 n-9$ & 19,5 & 19,3 & 18,8 \\
\hline $18: 1 n-7$ & 2,2 & 2,1 & 2,2 \\
\hline Mono-insaturés & 25,3 & 24,1 & 24,2 \\
\hline $18: 2 n-6$ & 4,0 & 3,0 & 4,1 \\
\hline $18: 3 n-3$ & 1,0 & 0,8 & 0,8 \\
\hline Polyinsaturés $(P)$ & 5,0 & 3,8 & 4,9 \\
\hline P/S & 0,07 & 0,05 & 0,07 \\
\hline
\end{tabular}


Tableau V. Composition en acides gras des triglycérides et des phospholipides du lactosérum et du concentré lipoprotéique (en \% des esters isopropyliques).

Fatty acid composition of triglycerides and phospholipids of whey and lipoprotein precipitate (in \% of isopropyl esters).

Acides gras

$\frac{\text { Lactosérum }}{\text { Triglycérides Phospholipides }}$

Concentré lipoprotéique

Triglycérides Phospholipides

\begin{tabular}{|c|c|c|c|c|}
\hline $4: 0$ & 1,1 & - & 1,0 & - \\
\hline $6: 0$ & 0,6 & - & 0,7 & - \\
\hline $8: 0$ & 1,3 & - & 1,6 & - \\
\hline $10: 0$ & 4,4 & 0,5 & 4,7 & 0,6 \\
\hline $12: 0$ & 5,4 & 1,2 & 5,3 & 1,3 \\
\hline $14: 0$ & 15,4 & 6,5 & 14,5 & 6,6 \\
\hline $15: 0$ & 1,7 & 1,2 & 1,6 & 1,4 \\
\hline $16: 0$ & 32,5 & 24,5 & 33,0 & 25,0 \\
\hline $17: 0$ & 0,7 & 0,6 & 0,8 & 0,7 \\
\hline $18: 0$ & 11,0 & 12,8 & 11,3 & 13,0 \\
\hline $20: 0$ & - & 0,4 & - & 0,3 \\
\hline 22:0 & - & 2,5 & - & 2,1 \\
\hline 23:0 & - & 2,7 & - & 2,5 \\
\hline $24: 0$ & - & 1,8 & - & 1,2 \\
\hline Saturés linéaires & 74,1 & 54,7 & 74,5 & 54,7 \\
\hline iso 15 & 0,6 & 0,3 & 0,6 & 0,4 \\
\hline ante iso 15 & 0,8 & 0,5 & 0,7 & 0,5 \\
\hline iso 17 & 0,7 & 0,5 & 0,8 & 0,6 \\
\hline ante iso 17 & 0,6 & 0,5 & 0,7 & 0,6 \\
\hline Saturés ramifiés & 2,7 & 1,8 & 2,8 & 2,1 \\
\hline Saturés totaux (S) & 76,8 & 56,5 & 77,3 & 56,8 \\
\hline $14: 1$ & 1,1 & 1,1 & 1,0 & 0,6 \\
\hline $16: 1 \mathrm{n}-9$ & 1,5 & 1,3 & 1,5 & 1,4 \\
\hline $16: 1 n-7$ & 1,3 & 0,5 & 1,2 & 1,4 \\
\hline $17: 1$ & 0,2 & - & 0,5 & 0,4 \\
\hline $18: 1 n-9$ & 14,4 & 28,9 & 13,5 & 28,7 \\
\hline $18: 1 n-7$ & 2,2 & 2,1 & 2,3 & 2,1 \\
\hline Mono-insaturés & 20,7 & 34,0 & 20,0 & 34,6 \\
\hline $18: 2 n-6$ & 1,9 & 7,1 & 2,0 & 6,2 \\
\hline $18: 3 n-3$ & 0,6 & 0,7 & 0,7 & 1,2 \\
\hline $20: 3 n-6$ & - & 0,9 & - & 0,6 \\
\hline $20: 4 n-6$ & - & 0,8 & - & 0,6 \\
\hline Polyinsaturés $(P)$ & 2,5 & 9,5 & 2,7 & 8,6 \\
\hline $\mathrm{P} / \mathrm{S}$ & 0,03 & 0,17 & 0,03 & 0,15 \\
\hline
\end{tabular}


VI). Les phospholipides les plus importants sont la phosphatidyl-éthanolamine (PE) (31-34\% des phospholipides totaux), la phosphatidyl-choline (PC) (30-31\%), la sphingomyéline (15-18\%), le phosphatidylinositol (PI) $(12 \%)$ et la phosphatidyl-sérine (PS) $(8-9 \%)$. Les cardiolipides sont absents.

Comparativement à l'extrait lipidique total, les phospholipides présentent une proportion d'acides gras saturés moins élevée $(56,5-56,8 \%)$. Ils sont exempts d'acides gras à courte chaîne et contiennent peu d'acides gras à chaîne moyenne. Par contre, les acides gras saturés à longue chaîne $(22: 0,23: 0$ et $24: 0)$ représentent $5,8-7,0 \%$. Le taux d'acides gras ramifiés est d'environ $2 \%$. Les acides gras monoinsaturés $(34,0-34,6 \%)$ sont essentiellement représentés par l'acide oléique $(31,0-30,8 \%)$. La teneur en acides gras polyinsaturés est de 8,6 à $9,5 \%$, soit 4 fois plus élevée que dans les triglycérides. L'acide gras polyinsaturé majeur est l'acide linoléique $(6,2-7,1 \%)$. L'acide linolénique (18:3 n-3), l'acide eicosatrienoïque (20:3 n6 ) et l'acide arachidonique (20:4 n-6) sont peu abondants $(0,6$ à $1,2 \%$ ) (tableau V).

Les classes de phospholipides issues du lactosérum et du concentré lipoprotéique ont des compositions en acides gras voisines (tableau VII). La PE apparaît comme le phospholipide le plus insaturé. Le taux d'acides gras saturés est inférieur à $30 \%$. Les acides gras mono-insaturés, essentiellement l'acide oléique, représentent environ $56 \%$. La teneur en acides gras polyinsaturés est de 14,5 à $17,4 \%$ suivant la fraction considérée. L'acide linoléique est l'acide gras polyinsaturé majeur (10,9 à $13,7 \%)$ alors que l'acide linolénique représente de 2,1 à $2,4 \%$, le $20: 3$ n-6 de 0,6 à $0,7 \%$ et

Tableau VI. Composition en phospholipides du lactosérum et du concentré lipoprotéique. Phospholipid distribution in whey and lipoprotein precipitate.

Lactosérum

Concentré lipoprotéique

en $\mathrm{mg} / 100 \mathrm{~g}$ de matière sèche

$\begin{array}{lrr}\text { PE } & 112 & 341 \\ \text { PI } & 40 & 134 \\ \text { PS } & 26 & 97 \\ \text { PC } & 102 & 330 \\ \text { SPH } & 50 & 198\end{array}$

Pourcentage des phospholipides totaux

$\begin{array}{lrr}\text { PE } & 34 & 31 \\ \text { PI } & 12 & 12 \\ \text { PS } & 8 & 9 \\ \text { PC } & 31 & 30 \\ \text { SPH } & 15 & 18\end{array}$

PE : phosphatidyl-éthanolamine; PI : phosphatidyl-inositol; PS : phosphatidyl-sérine; PC : phosphatidyl-choline; SPH : sphingomyéline. 
Tableau VII. Composition en acides gras des principales classes de phospholipides du lactosérum et du concentré lipoprotéique (en \% des esters méthyliques).

Fatty acid composition of the main phospholipid classes of whey and lipoprotein precipitate (in \% of isopropyl esters).

\begin{tabular}{|c|c|c|c|c|c|}
\hline Acides gras & $P E$ & $P I$ & $P S$ & $P C$ & $S P H$ \\
\hline \multicolumn{6}{|c|}{ Lactosérum } \\
\hline $14: 0$ & 1,5 & 1,0 & - & 3,1 & 1,7 \\
\hline 15:0 & 0,4 & 0,5 & - & 1,2 & 0,9 \\
\hline $16: 0$ & 13,4 & 24,1 & 10,7 & 35,6 & 47,1 \\
\hline $17: 0$ & 0,8 & 0,9 & - & 0,9 & - \\
\hline 18:0 & 13,0 & 22,4 & 21,1 & 13,2 & 10,7 \\
\hline $20: 0$ & - & - & - & - & - \\
\hline 22:0 & - & - & - & - & 8,4 \\
\hline 23:0 & - & - & - & - & 7,9 \\
\hline $24: 0$ & - & - & - & - & 3,2 \\
\hline Saturés & 29,1 & 48,9 & 31,8 & 54,0 & 79,9 \\
\hline $16: 1$ & 2,4 & 1,9 & - & 2,1 & 1,0 \\
\hline $17: 1$ & 0,3 & 1,0 & - & - & - \\
\hline $18: 1$ & 53,7 & 36,9 & 54,6 & 34,5 & 15,4 \\
\hline Mono-insaturés & 56,4 & 38,8 & 54,6 & 36,4 & 16,4 \\
\hline $18: 2 n-6$ & 10,9 & 9,3 & 11,8 & 7,6 & 2,9 \\
\hline $18: 3 n-3$ & 2,1 & 0,6 & 1,8 & 1,3 & 0,8 \\
\hline $20: 3 n-6$ & 0,7 & - & - & - & - \\
\hline $20: 4 n-6$ & 0,8 & - & - & - & - \\
\hline Polyinsaturés & 14,5 & 11,2 & 13,6 & 8,9 & 3,7 \\
\hline \multicolumn{6}{|c|}{ Concentré lipoprotéique } \\
\hline $14: 0$ & 0,9 & 2,2 & - & 3,8 & 1,5 \\
\hline 15:0 & 0,3 & - & - & 1,5 & 0,7 \\
\hline $16: 0$ & 12,7 & 22,4 & 7,5 & 42,0 & 46,7 \\
\hline $17: 0$ & 0,8 & 1,0 & 1,0 & 0,8 & 1,3 \\
\hline 18:0 & 12,4 & 24,4 & 24,8 & 12,6 & 10,4 \\
\hline 20:0 & - & - & - & - & 1,0 \\
\hline $22: 0$ & - & - & - & - & 11,1 \\
\hline 23:0 & - & - & - & - & 9,5 \\
\hline 24:0 & - & - & - & - & 3,0 \\
\hline Saturés & 27,1 & 50,0 & 33,3 & 60,7 & 85,2 \\
\hline $16: 1$ & 1,8 & 2,6 & 1,5 & 1,7 & 1,4 \\
\hline $17: 1$ & 0,5 & - & - & 0,7 & - \\
\hline $18: 1$ & 53,2 & 41,9 & 53,3 & 30,5 & 10,8 \\
\hline Mono-insaturés & 55,5 & 44,5 & 54,8 & 33,1 & 12,2 \\
\hline $18: 2 n-6$ & 13,7 & 5,5 & 9,9 & 5,3 & 2,3 \\
\hline $18: 3 n-3$ & 2,4 & - & 1,9 & 1,1 & 0,3 \\
\hline $20: 3 n-6$ & 0,6 & - & - & - & - \\
\hline $20: 4 n-6$ & 1,0 & - & - & - & - \\
\hline Polyinsaturés & 17,4 & 5,5 & 11,8 & 6,4 & 2,6 \\
\hline
\end{tabular}

PE: phosphatidyl-éthanolamine; PI: phosphatidyl-inositol; PS phosphatidyl-sérine; PC: phosphatidyl-choline; SPH: sphingomyéline. 
l'acide arachidonique atteint pratiquement 1\%. La PC est peu insaturée. Elle possède une forte proportion d'acides gras saturés $(54,0$ à $60,7 \%)$. Le taux d'acides gras mono-insaturés est de 33,1 à $36,4 \%$. Les acides gras polyinsaturés représentent de 6,4 à $8,9 \%$. Ce sont le $18: 2 n-6(5-7 \%)$ et le 18:3 n-3 (1,1-1,3\%). La sphingomyéline est le plus saturé des phospholipides. Les acides gras saturés représentent de 79,9 à $85,2 \%$. L'acide gras saturé majeur est le $16: 0(46,7-47,1 \%)$. On note la présence d'acides gras à chaîne longue en 22:0, 23:0 et 24:0 en quantité importante. Le 22:0 représente de 8,4 à $11,1 \%$, le $23: 0$ de 7,9 à $9,5 \%$ et le $24: 03,0-3,2 \%$. Les taux d'acides gras mono-insaturés et d'acides gras polyinsaturés sont respectivement de 12,2 à $16,4 \%$ et de 2,6 à $3,7 \%$.

La PS apparaît un peu plus insaturée que le PI. Le PI contient 48,9-50,0\% d'acides gras saturés, $38,8-44,5 \%$ d'acides gras mono-insaturés et $5,5-11,2 \%$ d'acides gras polyinsaturés. La PS contient $31,8-$ $33,3 \%$ d'acides gras saturés, $54,6-54,8 \%$ d'acides gras mono-insaturés et 11,8$13,6 \%$ d'acides gras polyinsaturés.

\section{DISCUSSION}

\section{Devenir des lipides au cours de la clarification du lactosérum}

Les compositions chimiques du lactosérum et de ses dérivés (concentré lipoprotéique et microfiltrat) sont comparables à celles rapportées antérieurement par Fauquant et al (1985a et b) et Baumy et al (1990). Ces résultats confirment que le procédé de clarification proposé par Fauquant et al (1985a et b) élimine une grande partie des lipides du lactosérum puisque les trois quarts des lipides initialement présents dans le lactosérum se retrouvent dans le concentré lipo- protéique et que seulement $8 \%$ restent dans le microfiltrat. Cependant, si le concentré est une fraction très enrichie en lipides, elle contient 7 fois plus de protéines que de lipides confirmant les travaux de Baumy et al (1990) ; 18\% des lipides du lactosérum sont perdus au cours du procédé. Baumy et al (1990) soulignent qu'une partie des lipides est éliminée lors de l'épuration bactérienne qui précède la clarification. Le reste des lipides se fixerait sur la membrane lors de la séparation microfiltrat / concentré lipoprotéique. Cette adsorption des lipides sur la membrane serait une des causes possibles de la réduction du débit de perméat lors de la microfiltration.

Nos résultats montrent que les lipides du lactosérum et du concentré lipoprotéique se composent d'environ deux tiers de triglycérides et un tiers de lipides polaires confirmant les données publiées par Baumy et al (1990) sur le lactosérum et plusieurs de ses coproduits et par Ducruet et al (1990) sur un concentré lipoprotéique. Les compositions des fractions phospholipidiques du lactosérum et du concentré lipoprotéique rapportées dans ce travail sont voisines de celles publiées par ces mêmes auteurs. Toutefois, selon nos résultats, ces lipides polaires sont pratiquement exclusivement composés de phospholipides, les céramides n'étant présents qu'en très faible proportion (moins de $2 \%$ de lipides polaires). Si Baumy et al (1990) ne signalent pas la présence de céramides dans la fraction lipides polaires, Ducruet et al (1990) indiquent qu'ils représentent plus d'un tiers des lipides polaires du concentré lipoprotéique. C'est pourquoi nous avons recherché de façon plus spécifique la présence de céramides dans nos échantillons. En procédant à une extraction des lipides selon la méthode de Marmer et Maxwell (1981) comme lavaient fait Ducruet et al (1990) et en déterminant les temps d'élution du lactosyl-céramide et du glucosyl-céramide dans 
nos conditions chromatographiques à l'aide de standards, nous n'avons observé que des pics mineurs au temps d'élution de ces composés. Par conséquent, l'explication de limportante différence entre nos résultats et ceux de Ducruet et al (1990) est à rechercher dans les difficultés de séparation et d'identification des classes de lipides polaires en couche mince. En effet, parallèlement à cette forte proportion de céramides mono et dihexosides (34\%), Ducruet et al (1990) n'ont mis en évidence qu'une faible proportion de PE $(9 \%$ de lipides polaires). Or, tous les travaux publiés à ce jour donnent des proportions inverses dans les produits laitiers ( 30 à $35 \%$ de $\mathrm{PE}$ et 1 à $8 \%$ de céramides) (Morrison, 1970; Jensen, 1973 ; Christie et al, 1987). Par ailleurs, Ducruet et al (1990) indiquent que les spots de PE et de céramides di-hexosides sont inversés sur la plaque par rapport aux positions décrites dans les travaux de Morrison et al (1965), qui avaient pourtant utilisé le même système de solvant. Cet ensemble d'observations nous conduit à penser que les céramides sont peu abondants dans le lactosérum et ses dérivés.

Sur la base des paramètres lipidiques considérés dans cette étude, il est possible de conclure que les compositions lipidiques du lactosérum et du concentré lipoprotéique sont similaires et, par conséquent que le procédé de clarification du lactosérum par agrégation thermocalcique n'exerce aucune ségrégation au sein de la fraction lipidique.

Bien que cette étude ne fournisse pas d'information directe sur les mécanismes de la clarification, elle permet de formuler l'hypothèse que la clarification du lactosérum résulterait d'une simple agrégation des globules gras de petites tailles avec les protéines sériques en présence du calcium et sous l'effet du traitement thermique. Le premier élément qui supporte cette hypothèse est la valeur comparable du rapport triglycérides / phospholipides des extraits lipidiques du concentré lipoprotéique et du lactosérum dont il est issu (deux tiers de triglycérides, un tiers de phospholipides). Le second réside dans le fait que ce ratio est proche de celui observé dans le lait écrémé et le lactosérum (Huang et Kuksis, 1967) dans lesquels les lipides se trouvent sous forme de petites vésicules (petits globules gras et/ou fragments membranaires) (Patton et Jensen, 1975 ; Pitot, 1984).

\section{Phospholipides du lactosérum}

Outre la clarification du lactosérum, le procédé développé par Fauquant et al (1985a, b) permet l'obtention d'une fraction enrichie en lipides. Si le concentré lipoprotéique contient peu de lipides ( $3 \%$ de la matière sèche), il serait possible d'accroître la teneur en lipides de façon très simple en éliminant les minéraux et le lactose par diafiltration. Un tel procédé a déjà été utilisé et permet d'obtenir des concentrés lipoprotéiques contenant jusqu'à $25 \%$ de lipides (Baumy et al, 1990). La mise en œuvre d'une telle technologie suppose que les concentrés trouvent une valorisation originale.

Les phospholipides du lactosérum et du concentré se composent principalement de phosphatidyl-éthanolamine $(30-34 \%)$, de phosphatidyl-choline $(30-31 \%)$, de sphingomyéline (15-18\%), de phosphatidyl-inositol $(12 \%)$ et de phosphatidyl-sérine $(8-9 \%)$. Cette répartition en classes des phospholipides est typique des produits laitiers (Jensen, 1973 ; Christie et al, 1987). Toutefois, la proportion de sphingomyéline dans le lactosérum et le concentré lipoprotéique de cette étude est légèrement inférieure à celle mesurée par d'autres auteurs dans des produits comparables (Baumy et al, 1990 ; Ducruet et al ,1990) ou dans le lait (Jensen, 1973 ; Christie et al, 1987) (15-18\% au lieu de $25-35 \%$ ). 
La composition en acides gras des phospholipides se caractérise par l'absence d'acides gras à chaîne courte et une teneur faible en acides gras polyinsaturés ( 8 à $9 \%$ ). Ces phospholipides ne contiennent pratiquement pas d'acides gras polyinsaturés à chaîne longue. La PE est le phospholipide le plus insaturé alors que la sphingomyéline est très saturée ( 80 à $85 \%$ des acides gras totaux). Ces résultats sont en accord avec ceux publiés antérieurement sur les phospholipides du lait (Morrison, 1970 ; Jensen, 1973) et des concentrés lipoprotéiques (Ducruet et al, 1990). Cependant, comparativement à nos résultats, Ducruet et al (1990) ont mis en évidence une proportion beaucoup plus élevée d'acides gras saturés à chaîne longue dans la sphingomyéline du concentré lipoprotéique. Cette différence pourrait être la conséquence d'une méthylation incomplète de ces acides gras dans nos conditions opératoires $\left(1 \mathrm{~h}\right.$ à $\left.100^{\circ} \mathrm{C}\right)$. En effet, Ducruet et al (1990) ont procédé à une méthylation de $14 \mathrm{~h}$ à $90^{\circ} \mathrm{C}$ en présence de méthanol / BF3.

Comparativement aux autres sources de phospholipides disponibles sur le marché, les phospholipides du lait tirent leur originalité de la présence d'une proportion importante de sphingomyéline et de leur faible proportion en acides gras polyinsaturés.

Si ce faible taux d'acides gras polyinsaturés peut nuire à l'image nutritionnelle de ces lipides, il constitue un atout dans le domaine technologique dans la mesure où ces phospholipides seront peu sensibles à l'oxydation. À notre connaissance, les propriétés fonctionnelles de la sphingomyéline n'ont pas été étudiées de sorte qu'il est impossible de se prononcer sur l'intérêt technologique de ce phospholipide. Cependant, cette composition phospholipidique des lipides du lait est voisine de celle des cellules de l'épiderme humain (Ziboh et Chapkin, 1988). Bien que la signification biologique de la présence d'une forte pro- portion de sphingomyéline dans les lipides de la peau ne soit pas établie, la cosmétologie pourrait constituer un débouché possible pour les phospholipides du lactosérum.

\section{CONCLUSION}

Cette étude montre que le procédé de clarification du lactosérum par agrégation thermocalcique des lipides développée par Fauquant et al (1985 a,b) permet un apurement presque total des lipides du lactosérum.

Quel que soit le coproduit considéré, la fraction lipidique se compose d'environ un tiers de phospholipides et de 2 tiers de triglycérides. Cette composition suggère que le procédé de clarification provoque l'agrégation des vésicules lipoprotéiques du lactosérum avec les protéines sériques sous l'effet de l'ajout de calcium et du traitement thermique.

L'originalité de la fraction lipidique du lactosérum réside dans sa composition phospholipidique. Les phospholipides du lactosérum et de ses dérivés se caractérisent par une faible teneur en acides gras polyinsaturés et par leur richesse en sphingomyéline.

\section{REMERCIEMENTS}

Les auteurs remercient vivement les chercheurs du laboratoire de technologie laitière de l'INRA de Rennes qui ont été à l'origine de ces travaux et la coopérative laitière de la région nantaise (Colarena, Herbignac) quí nous a fourni gracieusement les échantillons.

\section{REFERENCES}

Bartlett G (1959) Phosphorus assay in column chromatography. J Biol Chem $234,466-468$ 
Baumy JJ, Gestin L, Fauquant J, Boyaval E, Maubois JL (1990) Technologies de purification des phospholipides du lactosérum. Process 1047, 29-33

Blakeney AB, Harris PJ, Henry RJ, Stone BA (1983) A simple and rapid preparation of alditol acetates for monosaccharide analysis. Carbohydrate Res 113 , 291-299

Cerbulis J, Woychik JH, Wondolowski MV (1972) Composition of commercial wheys. J Agric Food Chem 20, 1057-1059

Christie WW, Noble RC, Davies G (1987) Phospholipids in milk and dairy products. J Soc Dairy Technol 40, 10-12

Clark RM, Ferris AM, Fey M, Brown PB, Hundrieser KE, Jensen RG (1982) Changes in the lipids of human milk from 2 to 16 weeks postpartum. J Pediatr Gastroenterol Nutr 1, 311-315

Ducruet V, Degas C, Lamberet C (1990) Composition lipidique d'un sous produit de lactosérum de caséinerie. Lait 70, 117-126

Fauquant J, Pierre A, Brulé G (1985a) Clarification du lactosérum acide de caséinerie. Tech Lait 1003, 37-41

Fauquant J, Vieco E, Brulé G, Maubois JL (1985b) Clarification des lactosérums doux par agrégation thermocalcique de la matière grasse résiduelle. Lait $65,1-20$

Huang TC, Kuksis A (1967) A comparative study of the lipids of globule membrane and fat core and of the milk serum of cows. Lipids 2, 453-460

Jensen RG (1973) Composition of bovine milk lipids. $J$ Am Oil Chem Soc 50, 186-192

Juaneda P, Rocquelin G (1985) Rapid and convenient separation of phospholipids and no phosphorus lipids from rat heart using silica cartridges. Lipids 20, 40-41

Leseigneur A, Gandemer G, Marion D (1989) Fractionnement en classes des lipides alimentaires par HPLC à l'aide d'un détecteur à diffusion de lumière. In: Actes du Congrès International Chevreul pour l'Etude des Corps Gras, édité par ETIG, Tome $1,311-318$

Marmer WN, Maxwell RJ (1981) Dry column method for quantitative extraction and simultaneous class separation of lipids from muscle tissue. Lipids 16, 365 371

Maubois JL, Pierre A, Fauquant J, Piot M (1987) Industrial fractionation of main whey proteins. Bull int Dairy Fed 212, 154-159

Mordret F, Coustille JL, Taconne L (1984) Dosage rapide du cholestérol dans les margarines. Rev $\mathrm{Fr}$ Corps Gras 31, 503-507

Morrison WR (1970) Milk Lipids. In: Topics in lipid chemistry, Vol 1, (Gunstone FD, ed) Logos Press, London, 51-106

Morrison WR, Smith LM (1964) Preparation of fatty acid methyl esters and dimethylacetals from lipids with boron-fluoride methanol. J Lipid Res 5, 600-608

Morrison WR, Jack EL, Smith LM (1965) Fatty acids of bovine milk glycolipids and phospholipids and their specific distribution in the diacylglycerophospholipids. J Am Oil Chem Soc 42, 1142-1147

Patton S, Jensen RG (1975) Lipid metabolism and membrane functions of the mammary gland. In: Progress in chemistry of fats and other lipids, vol 14 (Holman RT, ed), Pergamon Press Inc, London, 163-277

Pitot P (1984) Incorporation de lipides de sérum dans les crèmes de consommation : influence sur leurs propriétés fonctionnelles. Mémoire de fin d'étude ENSFA Rennes

Théodet C, Gandemer G (1991) Comparaison de 5 méthodes pour extraire les lipides du lactosérum et de ses dérivés. Lait 71, 41-54

Wolff RL, Fabien RJ (1989) Utilisation de l'isopropanol pour l'extraction de la matière grasse de produits laitiers et pour l'estérification subséquente des acides gras. Lait 69, 33-46

Ziboh VA, Chapkin RS (1988) Metabolism and function of skin lipids. Prog Lipid Res 27, 81-105 